modify steps in the partner services algorithm tested in this study to improve efficiency. In the meantime, application of the partner services algorithm as tested in this study seems to pose little risk because the new approach resulted in an almost 15 times increase in new testing and five times increase in new diagnoses. With effect sizes this large, a very large unknown confounder would have to be present in the environment for the findings not to be valid. Despite achievements to date and known barriers that have prevented adoption of partner services, findings from this study support calls to say no to complacency made during the AIDS 2016 conference in Durban. ${ }^{9}$ Scaling up of immediate assisted partner services is clearly an innovative casefinding approach that might be instrumental in helping countries achieve UNAIDS Fast-Track Targets by $2030 .{ }^{10}$

Some issues pertinent to sustainability exist. For example, the authors note counsellors' training requirements and suggest that some efforts might be needed to rely on "task shifting to a less highly educated cadre than those used in this study" in the future. Additionally, districts with administrative hurdles or low numbers of people with HIV infections were excluded from the trial. A national programme would of course have to overcome these hurdles and provide a minimum level of services for low-prevalence sites. That noted, the study incorporated 18 of 28 districts across Kenya with a-priori administrative acceptance, indicating first steps toward sustainability. In the USA, HIV partner services appear to be a costeffective prevention and control strategy. ${ }^{11}$ In countries such as many of those in sub-Saharan Africa, even though the cost of treatment might be lower than in the USA, and therefore cost-effectiveness harder to achieve, the number of undiagnosed HIV cases in subSaharan Africa suggests that any method that yields as many cases as shown in this study is likely to be worth the investment. For that matter, a partner services programme also reveals networks of transmission and social contact and connects people infected with HIV to life-saving health services. A well known comment in partner services literature reads "contact tracing's price is not its value", ${ }^{12}$ an axiom shown once again.

\section{*Matthew Hogben, Stephanie Behel}

Division of Sexually Transmitted Disease Prevention (MH) and Division of Global HIV and Tuberculosis (SB), Centers for Disease Control and Prevention, Atlanta, GA 30333, USA

mhogben@cdc.gov

We declare no competing interests

1 Joint UN Programme on HIV/AIDS. Global AIDS update. Geneva: Joint United Nations Programme on HIV/AIDS, 2016.

2 Joint UN Programme on HIV/AIDS. Prevention gap report. Geneva: Joint United Nations Programme on HIV/AIDS, 2016

3 Centers for Disease Control and Prevention. Recommendations for partne services programs for HIV infection, syphilis, gonorrhea, and chlamydial infection. MMWR Recomm Rep 2008; 57 (RR-9): 1-83.

4 Bernstein KT, Stephens SC, Moss N, Scheer S, Parisi MK, Philip SS Partner services as targeted HIV screening-changing the paradigm. Public Health Rep 2014; 129: 50-55.

5 Brown LB, Miller WC, Kamanga G, et al. HIV partner notification is effective and feasible in sub-Saharan Africa: opportunities for HIV treatment and prevention. J Acquir Immune Defic Syndr 2015; 56: 437-42.

6 Alam N, Chamot E, Vermund SH, Streatfield K, Kristensen S. Partner notification for sexually transmitted diseases in developing countries: a systematic review. BMC Public Health 2010; 10: 19.

7 Cherutich P, Golden MR, Wamuti B, et al, for the aPS Study Group. Assisted partner services for HIV in Kenya: a cluster randomised controlled trial. Lancet HIV 2016; published online Nov 29. http://dx.doi.org/10.1016/ S2352-3018(16)30214-4.

8 Aral SO, Blanchard JF. The Program Science initiative: improving the planning, implementation and evaluation of HIV/STI prevention programs. Sex Transm Infect 2012; 88: 157-59.

9 Joint UN Programme on HIV/AIDS. The 2016 International AIDS Conference closes with a call to reject complacency in the AIDS response. July 25, 2016. http://www.unaids.org/en/resources/presscentre/ featurestories/2016/july/20160722_aids2016closing (accessed Oct 19, 2016)

10 Joint UN Programme on HIV/AIDS. Fast-track. Ending the AIDS epidemic by 2030. Geneva: Joint United Nations Programme on HIV/AIDS, 2014

11 Lin F, Farnham PG, Shrestha RK, Mermin J, Sansom SL. Cost-effectiveness of HIV prevention programs in the U.S. Am J Prev Med 2016; 50: 699-708.

12 Potterat JJ. Contact tracing's price is not its value. Sex Transm Dis 1997; 24: 519-21.

\title{
Assessing progress with HIV incidence in national cohorts
}

Published Online November 15, 2016 http://dx.doi.org/10.1016/ S2352-3018(16)30187-4 See Articles page e 83
In The Lancet HIV, Jessica Justman and colleagues ${ }^{1}$ provide HIV incidence rates for Swaziland from a national 6 month follow-up study, making an important contribution to understanding the dynamics of the HIV epidemic in this small southern African country with a population of 1.25 million people. 145 HIV seroconversions were observed in a cohort of
11232 HIV-negative individuals during 6086 personyears of observation, leading to an HIV incidence rate of 2.4 per 100 person-years.

At the national-level, reliable estimates of both prevalence and incidence are required to appreciate the changing dynamics of HIV infection. Although data for HIV prevalence are common and routinely 
available, accurate data for incidence are scarce. Very few prospectively measured HIV incidence rates are available, beyond HIV prevention trials, at the district, national, or regional level. Instead, mathematical models or laboratory assays for recent infection are most often used to calculate HIV incidence on the basis of data or samples from one or more seroprevalence surveys.

Although useful, estimates from mathematical models should be interpreted with care as their results can vary substantially depending on both the structure of the model and assumptions involved. Several laboratory techniques, including the assay for p24 antigen in the absence of antibodies, the BED-CEIA assay (a capture enzyme immunoassay with gp41 peptides from HIV subtypes B, E, and D), and more recently, nucleic acid amplification in the absence of antibodies, can identify those recently infected to estimate HIV incidence. Even though the accuracy of tests has improved over the past decade, the variability in each test ${ }^{2}$ makes extrapolation dependent on what constitutes recent for each assay. Swaziland's closest neighbours have national HIV incidence estimates as determined with BED-CEIA assays (South Africa ${ }^{3}$ and Botswana ${ }^{4}$ ) or mathematical models (Zimbabwe ${ }^{5}$ and Mozambique ${ }^{6}$ ); none have cohort-based national HIV incidence measures. So, are national-based cohort studies to measure HIV incidence required?

Given that the UN has adopted the goal of ending AIDS as a global health threat by 2030, accurate estimates of HIV incidence over time will be needed to assess progress towards the attainment of this goal. To assess changes in HIV incidence, individual measures will need to be reliable, with narrow confidence intervals. Such measures of HIV incidence are going to become a key marker for assessing country-level HIV epidemic trends. Accurate measurements of incidence are also needed to measure the effectiveness of prevention interventions, either singly or in combination. Although cohort-based HIV incidence is routinely measured in randomised controlled trials assessing HIV prevention modalities, it is rarely used to assess the effect of national prevention programmes.

The main reason for the dearth of national, cohortbased HIV incidence measurements is that large cohorts need to be followed for long periods to get reliable estimates, making measurement of HIV incidence time consuming, expensive, and logistically and ethically difficult. ${ }^{8} \mathrm{~A}$ further limitation of cohort studies is that their provision of safer sex interventions such as HIV counselling, condom promotion, sexually transmitted infection treatment and other HIV prevention interventions might change the HIV risk behaviour of participants. Individuals might also modify their risk taking behaviour in response to their awareness of being observed, the so-called Hawthorne effect. ${ }^{9}$ As a result, there are concerns that measuring HIV incidence through identifying seroconversions during long-term follow-up might not produce accurate estimates of the HIV incidence.

Despite these limitations, the measurement of HIV seroconversions during follow-up is the gold standard for HIV incidence. Without cohort-based estimates, country-level progress toward the UN 2030 goal will be difficult to assess. As HIV incidence usually declines in small decrements, extrapolations from mathematical models or laboratory assays might not identify these changes because of the inherent variability in their estimates. Although multilevel sampling strategies are well established to provide reasonably representative samples, large studies will be required for cohortbased approaches to detect small changes in HIV incidence over time. In Swaziland, which is smaller than many cities, it was practically feasible to establish a national cohort to assess a national male circumcision intervention. ${ }^{1}$ This might be a much more complex task in larger countries.

Swaziland is the first country in southern Africa to have a national cohort-based HIV incidence rate, serving as an example to encourage others to follow suit. Accurate measurement of HIV incidence is taking

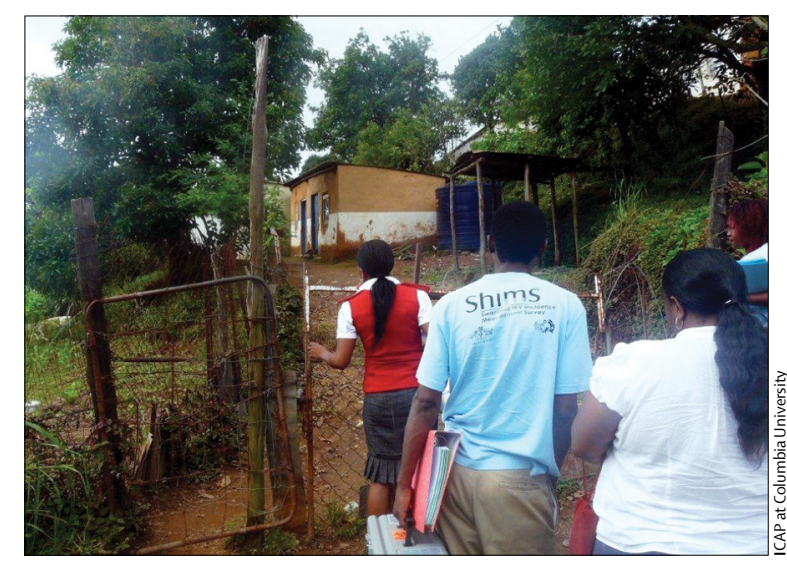


on a new level of importance for measuring the effect of population-level interventions and monitoring progress on the path to HIV epidemic control.

\section{Salim S Abdool Karim}

Centre of AIDS Programme of Research in South Africa (CAPRISA), Durban, South Africa; and Department of Epidemiology, Columbia University, New York, USA

salim.abdoolkarim@caprisa.org

I declare no competing interests

1 Justman J, Reed JB, Bicego G, et al. Swaziland HIV Incidence Measurement Survey (SHIMS): a prospective national cohort study. Lancet HIV 2016; published online Nov 15. http://dx.doi.org/10.1016/S2352-3018(16)30190-4

2 Kassanjee R, Pilcher CD, Keating SM, et al. Independent assessment of candidate HIV incidence assays on specimens in the CEPHIA repository. AIDS 2014; 28: 2439-49.
3 Shisana O, Rehle T, Simbayi LC, et al. South African national hiv prevalence, incidence, and behaviour survey 2012. Cape Town: HSRC Press, 2014.

4 Statistics Botswana. Botswana AIDS Impact survey IV (BAIS IV). http://www.cso.gov.bw/images/aids_summary.pdf (accessed Sept 8, 2016).

5 Mugurungi $\mathrm{O}$, Magure T, Mhangara M, et al. Zimbabwe National HIV and AIDS Estimates 2013. Harare, Zimbabwe: AIDS \& TB Programme Ministry of Health and Child Care, 2014

6 Perez-Hoyos S, Naniche D, Macete E, et al. Stabilization of HIV incidence in women of reproductive age in southern Mozambique. HIV Medicine 2011; 12: $500-05$

7 UNAIDS. 90-90-90 An ambitious treatment target to help end the AIDS epidemic. http://www.unaids.org/en/resources/ documents/2014/90-90-90 (accessed March 12, 2016).

8 Gouws E. HIV incidence rates in South Africa. In: Abdool Karim S, Abdool Karim Q, eds. HIV/AIDS in South Africa, 2nd edn. Cape Town: Cambridge University Press, 2010: 74-84

9 Padian NS, McCoy SI, Balkus JE, Wasserheit JN. Weighing the gold in the gold standard: chal lenges in HIV prevention research. AIDS 2010; 24: 621-35. 